\title{
Influence of healthy candidate bias in assessing clinical effectiveness for implantable cardioverter-defibrillators: cohort study of older patients with heart failure
}

\author{
(c) $(1)$ (8)
}

\author{
Soko Setoguchi associate professor ${ }^{12}$, Lynne Warner Stevenson professor ${ }^{3}$, Garrick C Stewart \\ instructor $^{3}$, Deepak L Bhatt professor ${ }^{3}$, Andrew E Epstein professor ${ }^{4}$, Manisha Desai associate \\ professor $^{5}$, Lauren A Williams research assistant ${ }^{1}$, Chih-Ying Chen postdoctoral fellow ${ }^{1}$
}

'Division of Pharmacoepidemiology and Pharmacoeconomics, Brigham and Women's Hospital and Harvard Medical School, Boston, MA, USA; ${ }^{2}$ Duke Clinical Research Institute, Duke University School of Medicine, Durham, NC 27715, USA; ${ }^{3}$ Division of Cardiovascular Medicine, Brigham and Women's Hospital and Harvard Medical School, MA, USA; ${ }^{4}$ Electrophysiology Section, Cardiovascular Division, University of Pennsylvania, Philadelphia, PA, USA; ${ }^{5}$ Quantitative Sciences Unit, Stanford University School of Medicine, Palo Alto, CA, USA

\begin{abstract}
Objective To assess the potential contribution of unmeasured general health status to patient selection in assessments of the clinical effectiveness of implantable cardioverter-defibrillator (ICD) therapy.

Design Retrospective cohort study.

Setting Linked data from an ICD registry, heart failure registry, and Medicare claims data for ICDs implanted in 2005 through 2009.

Participants 29426 patients admitted to hospital with heart failure aged 66 years or older and eligible for ICD therapy for primary prevention.

Main outcome measures Non-traumatic hip fracture, admission to a skilled nursing facility, and 30 day mortality-outcomes unlikely to be improved by ICD therapy.

Results Compared with 17853 patients without ICD therapy, 11573 patients with ICD therapy were younger and had lower ejection fraction and more cardiac admissions to hospital but fewer non-cardiac admissions to hospital and comorbid conditions. Patients with ICD therapy had greater freedom from unrelated events after adjusting for age and sex: hip fracture (hazard ratio $0.77,95 \%$ confidence interval 0.64 to 0.92$)$, skilled nursing facility admission $(0.53,0.50$ to 0.55$)$, and 30 day mortality $(0.12,0.10$ to 0.15$)$.

Conclusions Lower risks of measured outcomes likely reflect unmeasured differences in comorbidity and frailty. The findings highlight
\end{abstract}

potential pitfalls of observational comparative effectiveness research and support physician consideration of general health status in selecting patients for ICD therapy.

\section{Introduction}

After several landmark clinical trials showed the efficacy of implantable cardioverter-defibrillator (ICD) therapy, ${ }^{2}$ the US Centers for Medicare \& Medicaid Services (CMS) expanded coverage of ICD therapy to include primary prevention of sudden cardiac death in Medicare beneficiaries with heart failure. ${ }^{3}$ Medicare beneficiaries mostly consist of those aged 65 years or older, in whom the risk of sudden cardiac death is lower than in the trial patients..$^{4-6}$ None the less, one study projected that ICD therapy may be indicated for as many as 800000 additional people with heart failure in the United States, most of whom are 65 years or older. ${ }^{7}$

In real world modern clinical practice, patients are selected to receive ICD therapy on the basis of multiple factors, including indications and contraindications, underlying disease severity, comorbid conditions, and overall prognosis. Guideline recommendations for both heart failure and device based therapy include "anticipated survival of at least a year with good functional capacity," which is a crucial but subjective clinical 
assessment. In older patients, cognitive and functional capacity and social support can influence not only prognosis but also preferences for a device that prevents sudden cardiac death without improvement in quality of life. Information about these factors is often unavailable in large databases, including claims data, registries, and medical records, which affect the validity of assessing comparative effectiveness of ICD therapy in real world patients using these data.

To understand the contribution of unmeasured general health status to selection of patients for ICD therapy and potential bias in observational studies using registries and claims data, we compared three outcomes among patients with and without ICD therapy that can reflect general health status but are unlikely to be improved by ICD therapy: hip fracture, admission to a skilled nursing facility, and 30 day mortality.

\section{Methods}

\section{Data sources}

We conducted a retrospective cohort study of older patients with heart failure using the CMS ICD registry (2005-08); the National Cardiovascular Database Registry's ICD registry (2005-08); a national clinical registry of patients with heart failure aggregated from several quality improvement and accreditation programs, including the American Heart Association's Get With the Guidelines program (2005-08); and data files on Medicare institutional and non-institutional claims (2004-09) (see web extra appendix I).

We linked the combined ICD registry and the heart failure registry to Medicare claims data using four non-unique identifiers: date of birth, sex, admission date for implantation of the ICD, and provider ID, which is described in detail elsewhere. ${ }^{8}$ Briefly, we validated this linkage among the subset of 196923 patients who had a unique identifier in the ICD registry. Our linkage using non-unique identifiers yielded $98 \%$ specificity, $95 \%$ sensitivity, and $98 \%$ positive predictive value compared with the linkage method using both non-unique and unique identifiers.

\section{Study population, cohorts, and treatment status}

Our study population represented patients with heart failure who were 66 years or older and eligible for ICD therapy for primary prevention (see web extra appendix II for detailed information on the study population). We identified two cohorts of patients with and without ICD therapy in the linked databases (fig $1 \Downarrow$ ). Cohort 1 consisted of the population of Medicare beneficiaries who were linked to the ICD registry (areas A and $B$ in fig 1) and the population of Medicare beneficiaries who were linked to the heart failure registry but not with the ICD registry (area C). Cohort 2, a smaller but conceptually more appropriate study population, consisted of Medicare

beneficiaries who were linked to the heart failure registry with ICD therapy (area B) and without ICD therapy (area C). We conducted analyses in both cohorts to ensure that the findings were similar. All study patients had an ejection fraction of $35 \%$ or less. We excluded patients who had a history of cardiac arrest or sustained ventricular tachycardia. All patients were eligible for Medicare for one year before the index procedure or the index admission to hospital and were 66 years or older. We excluded patients who underwent both cardiac resynchronization therapy and ICD therapy, because biventricular pacing improves cardiac function and could improve patients' functional status, potentially reducing the risk of the outcomes of interest.

\section{Outcomes}

We selected three outcomes that are not causally associated with ICD therapy, are markers for frailty and other unmeasured pre-existing patient characteristics, and can be accurately measured with high specificity in Medicare data. These included admission for non-traumatic hip fracture (that is, fracture not due to trauma other than a fall and often a consequence of osteoporosis and a fall), admission to a skilled nursing facility (a licensed facility that provides short term or long term skilled care, commonly called a "nursing home"), and 30 day mortality. We identified admissions to hospital for non-traumatic hip fracture on the basis of a diagnosis of hip fracture (international classification of diseases, 9th revision, clinical modification (ICD-9-CM) diagnosis code 820.xx or 733.14) and a related procedure code (ICD-9-CM procedure code 78.55, 79.05, 79.15, $79.25,79.35$, or 79.65 or current procedural terminology (CPT) code $27230-27248) .{ }^{9}$ We identified admissions to a skilled nursing facility in the Medicare institutional files. Because some such skilled nursing facility admissions identified in Medicare data are short term and may not be totally related to frailty, we also identified a subset of nursing home admissions that resulted in stays of more than 20 days (beyond the period for full Medicare coverage) or death within 20 days as a more accurate indicator of frailty. We chose mortality within 30 days, a timeframe during which prophylactic ICD therapy did not provide a benefit in the landmark clinical trials. For example, the Sudden Cardiac Death in Heart Failure Trial (SCD-HeFT) found that the mortality benefit started to appear approximately 1.5 years after implantation of an ICD. ${ }^{1}$ All patients were followed until the occurrence of the outcomes and death from the index date, which was the date of implantation for those who received ICD therapy and the discharge date from their admission to hospital for heart failure for those who did not receive ICD therapy.

\section{Statistical analysis}

In patients with or without ICD therapy, we described baseline characteristics, including use of several preventive care and screening tests as markers for health seeking behaviors. We reported counts and person time for each outcome and graphed cumulative outcome event risks over time using Kaplan-Meier estimators. We used proportional hazards regression to derive crude and adjusted hazard ratios comparing patients with and without ICD therapy.

Some values were missing for systolic blood pressure and serum levels of sodium, B type natriuretic peptide, and creatinine in some patients (see table 1). We handled missing values by using multiple imputation ${ }^{10}$ and assuming an underlying multivariate normal distribution. Our analysis was based on five imputed datasets where the imputation model included all variables in the outcome model (ICD use outcomes and potential confounders) as well as variables related to missingness (see web extra appendix IV). ${ }^{11}$

\section{Sensitivity analyses}

We repeated the same analyses after excluding patients older than 80 years and those with a previous admission to a skilled nursing facility, because the indication criterion of "expected survival with good functional capacity for $>1$ year" is less likely to be met for these patients.

We used SAS version 9.2 (SAS Institute, Cary, NC) for all analyses. 


\section{Results}

We identified 29426 patients with heart failure (11 573 with ICD therapy and 17853 without ICD therapy) in cohort 1 who met the eligibility criteria. Patients who received ICD therapy were younger and more likely to be men and white than patients who did not receive ICD therapy. Patients with ICD therapy also had lower ejection fractions, more previous admissions to hospital for cardiac diseases, more physician visits, and ischemic etiology of heart failure (table $1 \Downarrow$ ). Patients without ICD therapy had a higher prevalence of non-cardiac admissions to hospital, previous admissions to a skilled nursing facility, more chronic kidney or lung disease, metastatic cancer, and other non-cardiovascular diseases. The prevalence of many preventive procedures and screening tests was higher in patients with ICD therapy. These findings were similar in cohort 2.

During 42580 person years of follow-up (mean, 1.4 years) in cohort 1 , we observed 676 admissions to hospital for hip fracture and 9475 admissions to a skilled nursing facility (table $2 \Downarrow$ ). During the first 30 days, 2428 deaths occurred. Patients without ICD therapy had substantially higher incidence rates of non-traumatic hip fracture (17 $v 9$ per 1000 person years), admissions to a skilled nursing facility (354 $v 112$ per 1000 person years), and 30 day mortality (1699 $v 165$ per 1000 person years).

The cumulative risk of hip fracture at three years was $4.8 \%$ (95\% confidence interval $4.3 \%$ to $5.3 \%$ ) among patients without ICD therapy and $2.6 \%$ (2.3\% to $3.1 \%$ ) among patients with ICD therapy. The cumulative incidence curves diverged immediately after implantation of the ICD (fig $2 \Downarrow$ ). The cumulative risk of admission to a skilled nursing facility at one year was $13.4 \%$ $(12.8 \%$ to $14.0 \%)$ with ICD therapy and $35.7 \%$ (34.9\% to $36.4 \%$ ) without ICD therapy. These curves diverged immediately and paralleled within a few days after implantation of the ICD (fig 2). The pattern was similar for 30 day mortality (fig 2). The findings were similar in cohort 2 (see web extra appendix V).

Unadjusted rates of outcomes in patients with ICD therapy were lower than in patients without ICD therapy for admission for non-traumatic hip fracture (hazard ratio $0.51,95 \%$ confidence interval 0.43 to 0.59 ), admission to a skilled nursing facility $(0.39,0.37$ to 0.41$)$, and 30 day mortality $(0.10,0.09$ to 0.12$)$ (table $3 \Downarrow$ ). After adjustment for age and sex, these estimates moved toward 1. After adjustment for all patient measured characteristics, these estimates moved further but only moderately toward 1 and the risks remained substantially lower among patients who received ICD therapy (table 3 ).

The refined outcome for admission to a skilled nursing facility, which used a stay of 20 days or longer or death within 20 days, yielded similar results (tables 2 and 3). After adjustment for all measured factors, the risks remained significantly lower among patients with ICD therapy. Restricting the cohorts to patients 80 years or younger (see web extra appendix VI) or with no previous admission to a skilled nursing facility (see web extra appendix VII) did not change the results meaningfully.

\section{Discussion}

We compared older patients with and without implantable converter-defibrillator (ICD) therapy for the risk of three outcomes that are unlikely to be directly improved by ICD therapy to assess the impact of unmeasured patient characteristics on patient selection for ICD therapy. We observed a $50 \%$ to $60 \%$ lower risk for admission for non-traumatic hip fracture, admission to a skilled nursing facility, and 30 day mortality in patients with ICD therapy compared with patients with heart failure without ICD therapy. These differences in the risks of unrelated outcomes were observed immediately after implantation of the ICD. The differences lessened after adjustment for measured patient characteristics, but risks remained lower in patients who received ICD therapy by $16 \%$ to $34 \%$. Given the timing and size of the observed differences, these results likely reflects baseline differences between the candidates for ICD therapy and patients who did not receive ICD therapy that are not measured in Medicare or registry data, leading to selection bias or "healthy candidate bias." From a clinical perspective, these differences reflect appropriate integration of multiple health dimensions into clinical decision making in modern clinical practice to select patients who are most likely to benefit.

The healthy candidate bias that we observed is analogous to biases described in other fields of epidemiology. Occupational epidemiologists have long recognized the "healthy worker survivor effect" when evaluating effects of environmental exposures. This bias arises in comparisons of workers exposed to work related toxins and workers not exposed because relatively healthy people are likely to gain employment and remain employed, whereas severely ill and chronically disabled people are ordinarily excluded from employment. ${ }^{12}$ Similar bias, known as "healthy user bias" in pharmacoepidemiology, has been observed among users of preventive medications and vaccines, such as hormone replacement therapy, ${ }^{13}{ }^{14}$ statins,,${ }^{15} 16$ and influenza vaccine. ${ }^{17}$ Because implantable devices and surgical procedures typically pose short term risks in exchange for long term benefits, patients at high risk of complications or deemed too sick to benefit are less likely to be selected. In the Cardiac Arrhythmia Suppression Trial, a similar effect was observed. ${ }^{18}$ This healthy candidate effect is one of the biggest threats to validity in observational comparative effectiveness research, especially in comparisons of invasive interventions to less invasive alternatives.

Frailty reflected by low functional status and impaired cognitive function, lack of social support, unrecognized or untreated depression, and diminished quality of life are associated with decreased survival in patients with heart failure. ${ }^{19-23}$ Each of these factors may be associated with the decision to offer and receive ICD therapy. For example, preference for survival was strongly associated with longer survival in patients with heart failure in the Evaluation Study of Congestive Heart Failure and Pulmonary Artery Catheter Effectiveness. ${ }^{24}$ Among patients who survived fewer than 105 days, $31 \%$ indicated that they would trade more than $90 \%$ of their survival days to feel well for the time remaining, compared with $6 \%$ of patients who survived all 180 days $(\mathrm{P}<0.005)$, indicating that those most likely to die were least likely to have cared about prolonging survival. ${ }^{24}$ In another survey study of 105 consecutive patients with mean ejection fraction of $21 \%, 65 \%$ of whom received ICD therapy for primary prevention, recipients of ICD therapy expressed greater confidence that the device would save their own lives than those without ICD therapy $(\mathrm{P}<0.001) .{ }^{25}$ These data indicate that recipients of ICD therapy tend to have a strong preference for survival, which is likely to correlate directly with lower rates of death, admission to a skilled nursing facility, and even hip fracture indirectly. Finally, incomplete depiction of non-cardiovascular comorbidity is likely another source of unmeasured differences between patients with and without ICD therapy. ${ }^{25} 26$ 


\section{Clinical and policy implications}

Eligibility criteria for ICD therapy from both the heart failure guidelines ${ }^{26-28}$ and the device based therapy guidelines ${ }^{29}{ }^{28}$ from the American College of Cardiology, the American Heart Association, and the European Society of Cardiology include anticipated survival with "good functional capacity" for at least one year. We found that $13 \%$ of patients with ICD therapy and $36 \%$ of patients without ICD therapy were admitted to skilled nursing facilities within a year if they did not die. Considering a composite outcome of admission to a skilled nursing facility and death, $55 \%$ of patients without ICD therapy would not meet the expectation of one year survival with good functional capacity, whereas a smaller fraction $(21 \%)$ of patients with ICD therapy would meet the expectation.

A few previous studies have concluded that ICD therapy may be underused in general or certain Medicare patients without specific contraindications to implantation of a ICD for primary prevention of sudden cardiac death. ${ }^{29-31}$ While existing cardiovascular registries and Medicare data include information on skilled nursing facilities and ICD-9-CM diagnosis codes for comorbid conditions, they lack documentation of the severity of comorbidity, socioeconomic factors, functional status, cognitive function, and quality of life. Therefore, apparent gaps in ICD use assessed using information available in registries and Medicare data may arise, at least in part, from incomplete depiction of the appropriateness of patient selection for ICD therapy.

Our results indicate that a substantial fraction of patients who were potentially eligible for ICD therapy but did not receive it may have been at high risk for not "surviving with good quality of life." However, our study had limited ability to identify patients who were specifically at higher risk for admission to a skilled nursing facility. There are without doubt patients who did not receive ICD therapy who would have derived meaningful life extension from prevention of sudden death, which are also not possible to identify in our analyses. Furthermore, some patients may have declined ICD therapy despite an appropriate recommendation.

\section{Implications for observational comparative effectiveness research}

Although observational studies provide data on real world patients in real world settings, they are subject to potential bias by selection, confounding, survivor bias, and inaccurate measurement of variables. ${ }^{31}{ }^{32}$ Confounding by indication is described well in the pharmacoepidemiology literature ${ }^{32}{ }^{33}$ and can cause significant bias. However, registries typically include information on indications and contraindications for treatments. Therefore, confounding by indication was not a problem in our study using linked registry and claims data. Healthy candidate bias, a type of selection or confounding bias, can be the most challenging bias in observational comparative effectiveness research for devices and surgeries. We showed the existence of substantial healthy candidate bias in our ICD example, which was not controlled by multivariable adjustments for measured factors and might have affected, at least in part, previous ICD effectiveness studies. ${ }^{33-35}$

Various design-based and statistical approaches have been suggested to overcome healthy worker survivor effect or healthy user bias. These include restriction to survivors, ${ }^{35}{ }^{36}$ lagged time or latency analysis, ${ }^{36}$ g-computation, ${ }^{37-39}$ and comparison to active users of similar preventive medications. ${ }^{3940}$ The technique of restriction to a subset of survivors or to more comparable groups is a standard approach in epidemiology but one that is sometimes overlooked or intentionally avoided in favor of a larger sample size. Other methods, including latency analysis and g-computation, should be considered and used when appropriate. Finally, adjustment for proxies for unmeasured factors by maximizing available information in large databases using appropriate methods (for example, high dimensional propensity score methods) can potentially achieve better control for bias in some settings. ${ }^{40} 41$

This study suggests two important implications. Firstly, those responsible for the allocation of scarce healthcare resources in any country must carefully evaluate whether the efficacy evidence of expensive technologies such as ICD therapy apply to their use outside of clinical trials. Secondly, evaluations of effectiveness from clinical and administrative databases may overestimate the benefit of device or surgical therapies for which selection is influenced by healthy candidate bias.

Several limitations should be considered in interpreting these findings. Firstly, we identified patients who received and did not receive ICD therapy from different registries, which limited our ability to adjust for some factors such as New York Heart Association functional classification that were only measured in one of the registries. However, all patients were admitted to hospital with heart failure, both registries had equally limited information on non-cardiovascular comorbid conditions, and neither registry included information on frailty or social support. Also, all patients were Medicare beneficiaries, which was the target population of our study, and the findings were replicated in the secondary cohort (cohort 2) identified in the heart failure registry. In the comparison of 30 day mortality, it is possible that there was early excess non-sudden death in the ICD arm, as reported after myocardial infarction. ${ }^{41}{ }^{42}$ However, this would only have served to diminish the noticeable excess of 30 day mortality observed among patients who did not receive ICD therapy. For the non-fatal outcomes, competing risks by death exist in the current study. However, if ICD survival benefit from the trials was translated into this population, our results would have been underestimates of the true differences in the occurrence of hip fracture and admissions to a skilled nursing facility, rendering the selection due to frailty and other unmeasured factors even larger than observed here.

\section{Conclusion}

Difference in survival between patients receiving and not receiving ICD therapy in this large observational study was influenced by unmeasured differences in baseline health status, as indicated by lower risk of non-traumatic hip fracture, admission to a skilled nursing facility, and 30 day mortality among recipients of ICD therapy. Our analyses of early mortality and non-fatal outcomes highlight potential pitfalls of observational comparative effectiveness analyses of outcomes associated with one treatment versus another due to healthy candidate bias. It is not possible to determine from this study the degree to which apparent underutilization of such therapies may instead reflect thoughtful clinical decisions in which doctors use factors such as frailty to allocate expensive therapies to patients who are most likely to benefit.

Harlan M Krumholz, Yale University, provided thoughtful comments on an early draft of the manuscript. Damon M Seils, Duke University, provided editorial assistance and helped prepare the manuscript for submission.

This work was presented in part at the Agency for Healthcare Research and Quality 2011 national conference, September 21, 2011, in Bethesda, 


\section{What is already known on this topic}

Clinical trials of implantable cardioverter-defribrillators (ICDs) under-represent older patients with comorbidities, in whom the risk of sudden death might be lower, but many ICDs have been used in the United States and other countries

In clinical practice, treatments are not randomly allocated but selected based on indications and other considerations

Unmeasured selection factors can influence estimates of comparative effectiveness in observational studies, as shown by the healthy worker effect or healthy user bias in occupational research and pharmacoepidemiology

\section{What this study adds}

Evaluations of effectiveness from clinical and administrative databases may overestimate the benefit of device or surgical therapies for which selection is influenced by healthy candidate bias

Apparent underutilization of ICDs in older patients may instead reflect thoughtful clinical decisions in clinical practice in which clinicians use factors such as frailty to allocate expensive therapies to patients who are most likely to benefit

Maryland, and at the 2013 scientific meeting for the American Heart Association, November 18, 2013, in Dallas, Texas.

Contributors: SS and LWS contributed to the acquisition of the data. SS and LWS developed the study design. SS, CYC, and MD contributed to the analysis of the data. SS and CYC drafted the manuscript. All authors contributed to the interpretation of the data and the revision of the work, and all approved the final version to be published. SS is guarantor. The authors of this report are responsible for its content. Statements in the report should not be construed as endorsement by the Agency for Healthcare Research and Quality or the US Department of Health and Human Services.

Funding: This project was funded under contract No HHSA29020050016I, task order 3, from the Agency for Healthcare Research and Quality, US Department of Health and Human Services, as part of the Developing Evidence to Inform Decisions about Effectiveness (DEcIDE) program; and contract No HHSM500201000001I, task orders 2 and 6, from the Centers for Medicare \& Medicaid Services, US Department of Health and Human Services. SS was supported by midcareer development award K02HS017731 from the Agency for Healthcare Research and Quality. Competing interests: All authors have completed the ICMJE uniform disclosure form at www.icmje.org/coi_disclosure.pdf (available on request from the corresponding author). SS reported receiving grants from Agency for Healthcare Research and Quality and from the Center for Medicare and Medicaid Services during the conduct of the study. She has made available online a detailed listing of financial disclosures (www.dcri.duke.edu/about-us/conflict-of-interest/). DLB reported receiving grants from Amarin, AstraZeneca, Bristol-Myers Squibb, Eisai, Ethicon, Medtronic, Roche, Sanofi Aventis, and The Medicines Company; conducting unfunded research for FlowCo, PLx Pharma, and Takeda; receiving personal fees from the Duke Clinical Research Institute, the Mayo Clinic, the Population Health Research Institute, the American College of Cardiology, Belvoir Publications, Slack Publications, WebMD, and Elsevier Practice Update Cardiology; serving on advisory boards for Elsevier Practice Update Cardiology, Medscape Cardiology, and Regado Biosciences; serving on the board of directors of the Boston VA Research Institute and the Society of Cardiovascular Patient Care; receiving non-financial support from the Society of Cardiovascular Patient Care and the American Heart Association; and serving as editor for the Journal of Invasive Cardiology, outside of the submitted work. AEE reported receiving honorariums from Biotronik, Boston Scientific, Medtronic, and St Jude Medical; receiving research funding from Biotronik, Boston Scientific, Medtronic, and St Jude Medical; and receiving fellowship program support from Biotronik, Boston Scientific, Medtronic, and St Jude Medical.

Ethical approval: This study was approved by the institutional review board of Brigham and Women's Hospital.

Data sharing: No additional data available.

Transparency: The lead author affirms that this manuscript is an honest, accurate, and transparent account of the study being reported; that no important aspects of the study have been omitted; and that any discrepancies from the study as planned (and, if relevant, registered) have been explained.

1 Bardy GH, Lee KL, Mark DB, Poole JE, Packer DL, Boineau R, et al. Amiodarone or an implantable cardioverter-defibrillator for congestive heart failure. N Engl J Med 2005;352:225-37.

2 Moss AJ, Zareba W, Hall WJ, Klein H, Wilber DJ, Cannom DS, et al. Prophylactic implantation of a defibrillator in patients with myocardial infarction and reduced ejection fraction. N Engl J Med 2002;346:877-83.

3 Centers for Medicare \& Medicaid Services. National Coverage Determination (NCD) for Implantable Automatic Defibrillators (20.4). 2013. www.cms.gov/medicare-coveragedatabase/details/ncd-details. aspx?NCDId=110\&ncdver $=3$.

4 Tung P, Albert CM. Causes and prevention of sudden cardiac death in the elderly. Nat Rev Cardiol 2013;10:135-42.

5 Mehta PA, Dubrey SW, McIntyre HF, Walker DM, Hardman SM, Sutton GC, et al. Mode of death in patients with newly diagnosed heart failure in the general population. Eur $J$ Heart Fail 2008;10:1108-16.

6 Setoguchi S, Nohria A, Rassen JA, Stevenson LW, Schneeweiss S. Maximum potential benefit of implantable defibrillators in preventing sudden death after hospital admission because of heart failure. CMAJ 2009;180:611-6.

7 Fonarow GC, Yancy CW, Hernandez AF, Peterson ED, Spertus JA, Heidenreich PA. Potential impact of optimal implementation of evidence-based heart failure therapies on mortality. Am Heart J 2011;161:1024-30.e3.

8 Setoguchi S, Zhu Y, Jalbert JJ, Williams LA, Chen CY. Validity of deterministic record linkage using multiple indirect personal identifiers: linking a large registry to claims data. Circ Cardiovasc Qual Outcomes 2014; published online 22 Apr.

9 Ray WA, Griffin MR, Fought RL, Adams ML. Identification of fractures from computerized medicare files. J Clin Epidemiol 1992;45:703-14.

10 Little RJ, Rubin DB. Statistical analysis with missing data. John Wiley, 2002.

$11 \mathrm{Knol} \mathrm{MJ}$, Janssen KJ, Donders AR, Egberts AC, Heerdink ER, Grobbee DE, et al. Unpredictable bias when using the missing indicator method or complete case analysis for missing confounder values: an empirical example. J Clin Epidemiol 2010;63:728-36.

$12 \mathrm{Li} \mathrm{CY}$, Sung FC. A review of the healthy worker effect in occupational epidemiology. Occup Med (Lond) 1999;49:225-9.

13 Hemminki E, Malin M, Topo P. Selection to postmenopausal therapy by women's characteristics. J Clin Epidemiol 1993;46:211-9.

14 Humphrey LL, Chan BKS, Sox HC. Postmenopausal hormone replacement therapy and the primary prevention of cardiovascular disease. Ann Intern Med 2002;137:273-84.

15 Glynn RJ, Schneeweiss S, Wang PS, Levin R, Avorn J. Selective prescribing led to overestimation of the benefits of lipid-lowering drugs. J Clin Epidemiol 2006;59:819-28.

16 Ray WA, Daugherty JR, Griffin MR. Lipid-lowering agents and the risk of hip fracture in a medicaid population. Inj Prev 2002;8:276-9.

17 Jackson LA, Jackson ML, Nelson JC, Neuzil KM, Weiss NS. Evidence of bias in estimates of influenza vaccine effectiveness in seniors. Int J Epidemiol 2006;35:337-44.

18 Hallstrom AP, Greene HL, Huther ML. The healthy responder phenomenon in non-randomized clinical trials. Stat Med 1991;10:1621-31.

19 Cacciatore F, Abete P, Mazzella F, Viati L, Della Morte D, D'Ambrosio D, et al. Frailty predicts long-term mortality in elderly subjects with chronic heart failure. Eur J Clin Invest 2005;35:723-30.

20 Frasure-Smith N, Lesperance F, Habra M, Talajic M, Khairy P, Dorian P, et al. Elevated depression symptoms predict long-term cardiovascular mortality in patients with atrial fibrillation and heart failure. Circulation 2009;120:134-40, 3p following 140.

21 Friedmann E, Thomas SA, Liu F, Morton PG, Chapa D, Gottlieb SS. Relationship of depression, anxiety, and social isolation to chronic heart failure outpatient mortality. Am Heart J 2006;152:940.e1-8.

22 Konstam V, Salem D, Pouleur H, Kostis J, Gorkin L, Shumaker S, et al. Baseline quality of life as a predictor of mortality and hospitalization in 5,025 patients with congestive heart failure. SOLVD Investigations. Studies of Left Ventricular Dysfunction Investigators. Am J Cardiol 1996;78:890-5.

23 Murberg TA. Long-term effect of social relationships on mortality in patients with congestive heart failure. Int J Psychiatry Med 2004;34:207-17.

24 Stevenson LW, Hellkamp AS, Leier CV, Sopko G, Koelling T, Warnica JW, et al. Changing preferences for survival after hospitalization with advanced heart failure. J Am Coll Cardiol 2008;52:1702-8.

25 Stewart GC, Weintraub JR, Pratibhu PP, Semigran MJ, Camuso JM, Brooks K, et al. Patient expectations from implantable defibrillators to prevent death in heart failure. $J$ Card Fail 2010;16:106-13.

26 Lee DS, Tu JV, Austin PC, Dorian P, Yee R, Chong A, et al. Effect of cardiac and noncardiac conditions on survival after defibrillator implantation. J Am Coll Cardiol 2007;49:2408-15.

27 Hunt SA, Abraham WT, Chin MH, Feldman AM, Francis GS, Ganiats TG, et al. ACC/AHA 2005 Guideline Update for the Diagnosis and Management of Chronic Heart Failure in the Adult: a report of the American College of Cardiology/American Heart Association Task Force on Practice Guidelines (Writing Committee to Update the 2001 Guidelines 
for the Evaluation and Management of Heart Failure): developed in collaboration with the American College of Chest Physicians and the International Society for Heart and Lung Transplantation: endorsed by the Heart Rhythm Society. Circulation 2005;112:e154-e235.

28 McMurray JJ, Adamopoulos S, Anker SD, Auricchio A, Böhm M, Dickstein K, et al. ESC Guidelines for the diagnosis and treatment of acute and chronic heart failure 2012: The Task Force for the Diagnosis and Treatment of Acute and Chronic Heart Failure 2012 of the European Society of Cardiology. Developed in collaboration with the Heart Failure Association of the ESC. Eur Heart J 2012;33:1787-847.

29 Epstein AE, Dimarco JP, Ellenbogen KA, Estes NA 3rd, Freedman RA, Gettes LS, et al. ACC/AHA/HRS 2008 guidelines for Device-Based Therapy of Cardiac Rhythm Abnormalities: executive summary. Heart Rhythm 2008;5:934-55.

30 Hernandez AF, Fonarow GC, Liang L, Al-Khatib SM, Curtis LH, LaBresh KA, et al. Sex and racial differences in the use of implantable cardioverter-defibrillators among patients hospitalized with heart failure. JAMA 2007:298:1525-32.

31 Piccini JP, Hernandez AF, Dai D, Thomas KL, Lewis WR, Yancy CW, et al. Use of cardiac resynchronization therapy in patients hospitalized with heart failure. Circulation 2008;118:926-33.

32 Bavry AA, Bhatt DL. Interpreting observational studies-look before you leap. J Clin Epidemiol 2006;59:763-4.

33 Salas M, Hotman A, Stricker BH. Confounding by indication: An example of variation in the use of epidemiologic terminology. Am J Epidemiol 1999;149:981-3.

34 Chan PS, Chow T, Kereiakes D, Schloss EJ, Waller T, Eagle K, et al. Effectiveness of implantable cardioverter-defibrillators in patients with ischemic heart disease and left ventricular dysfunction. Arch Intern Med 2006;166:2228-33.

35 Hernandez AF, Fonarow GC, Hammill BG, Al-Khatib SM, Yancy CW, O'Connor CM, et al. Clinical effectiveness of implantable cardioverter-defibrillators among Medicare beneficiaries with heart failure. Circ Heart Fail 2010:3;7-13.

36 Fox AC, Collier PF. Low mortality rates in industrial cohort studies due to selection for work and survival in the industry. Br J Prev Soc Med 1976;20:225-30.

37 Checkoway H, Pearce N, Hickey JL, Dement JM. Latency analysis in occupational epidemiology. Arch Environ Health 1990;45:95-100.
38 Robins J. A new approach to causal inference in mortality studies with a sustained exposure period: Application to control of the healthy worker survivor effect. Math Modeling 1986;7:1393-512.

39 Robins J. The control of confounding by intermediate variables. Stat Med 1989;8:679-701.

40 Setoguchi S, Glynn RJ, Avorn J, Mogun H, Schneeweiss S. Statins and the risk of lung, breast, and colorectal cancer in the elderly. Circulation 2007;115:27-33.

41 Schneeweiss S, Rassen JA, Glynn RJ, Avorn J, Mogun H, Brookhart MA. High-dimensional propensity score adjustment in studies of treatment effects using health care claims data. Epidemiology 2009;20:512-22.

42 Hohnloser SH, Kuck KH, Dorian P, Roberts RS, Hampton JR, Hatala R, et al. Prophylactic use of an implantable cardioverter-defibrillator after acute myocardial infarction. $N$ Engl $J$ Med 2004;351:2481-8.

43 Levey AS, Stevens LA, Schmid CH, Zhang YL, Castro AF 3rd, Feldman HI, et al. A new equation to estimate glomerular filtration rate. Ann Intern Med 2009;150:604-12.

44 Skali H, Uno H, Levey AS, Inker LA, Pfeffer MA, Solomon SD. Prognostic assessment of estimated glomerular filtration rate by the new chronic kidney disease epidemiology collaboration equation in comparison with the modification of diet in renal disease study equation. Am Heart J 2011;162:548-54.

\section{Accepted: 25 March 2014}

\section{Cite this as: BMJ 2014;348:g2866}

This is an Open Access article distributed in accordance with the Creative Commons Attribution Non Commercial (CC BY-NC 3.0) license, which permits others to distribute, remix, adapt, build upon this work non-commercially, and license their derivative works on different terms, provided the original work is properly cited and the use is non-commercial. See: http://creativecommons.org/licenses/by-nc/3.0/. 


\section{Tables}

Table 1| Measured baseline characteristics of patients with or without implantable cardioverter-defibrillator (ICD) therapy. Values are numbers (percentages) unless stated otherwise

\begin{tabular}{|c|c|c|c|c|}
\hline \multirow[b]{2}{*}{ Characteristics } & \multicolumn{2}{|c|}{ Cohort 1 (ICD+heart failure registry population) } & \multicolumn{2}{|c|}{ Cohort 2 (heart failure registry population) } \\
\hline & No ICD ( $n=17853)$ & ICD $(n=11573)$ & No ICD $(n=17853)$ & ICD (n=1089) \\
\hline Mean (SD) age (years) & $80.0(7.8)$ & $75.0(5.8)$ & $80.0(7.8)$ & $75.1(5.9)$ \\
\hline Men & $9321(52)$ & $8514(74)$ & $9321(52)$ & $770(71)$ \\
\hline White race & $15068(84)$ & $10035(87)$ & $15068(84)$ & $888(82)$ \\
\hline \multicolumn{5}{|l|}{ Previous admissions and outpatient visits: } \\
\hline$\geq 1$ heart failure admission & $2844(16)$ & $2196(19)$ & $2844(16)$ & $354(33)$ \\
\hline$\geq 1$ non-cardiac admission & $6501(36)$ & $3290(28)$ & $6501(36)$ & $318(29)$ \\
\hline$\geq 1$ SNF admission & $2679(15)$ & $663(6)$ & $2679(15)$ & $84(8)$ \\
\hline Mean (SD) outpatient visits & $10.3(9.4)$ & $12.7(8.8)$ & $10.3(9.4)$ & $13.0(8.7)$ \\
\hline \multicolumn{5}{|l|}{ Medical history: } \\
\hline \multicolumn{5}{|l|}{ Cancer } \\
\hline Any cancer* & $3020(17)$ & $1946(17)$ & $3020(17)$ & $177(16)$ \\
\hline Metastatic cancer & $566(3)$ & $161(1)$ & $566(3)$ & $17(2)$ \\
\hline Cerebrovascular disease & $3860(22)$ & $2150(19)$ & $3860(22)$ & $229(21)$ \\
\hline Chronic kidney disease & $8009(45)$ & $3819(33)$ & $8009(45)$ & $407(37)$ \\
\hline COPD & $8283(46)$ & $4908(42)$ & $8283(46)$ & $523(48)$ \\
\hline Dementia & $3503(20)$ & $947(8)$ & $3503(20)$ & $83(8)$ \\
\hline Depression & $2821(16)$ & $1282(11)$ & $2821(16)$ & $142(13)$ \\
\hline Diabetes mellitus & $8648(48)$ & $5698(49)$ & $8648(48)$ & $584(54)$ \\
\hline Dialysis & $527(3)$ & $246(2)$ & $527(3)$ & $26(2)$ \\
\hline Gastrointestinal ulcer or bleeding & $2755(15)$ & $1405(12)$ & $2755(15)$ & $131(12)$ \\
\hline Heart failure of ischemic origin & $14165(79)$ & $10028(87)$ & $14165(79)$ & $954(88)$ \\
\hline Liver disease & $1118(6)$ & $629(5)$ & $1118(6)$ & $73(7)$ \\
\hline Peripheral vascular disease & $4147(23)$ & $2591(22)$ & $4147(23)$ & $270(25)$ \\
\hline \multicolumn{5}{|l|}{ Clinical characteristics: } \\
\hline $\begin{array}{l}\text { Median (interquartile range) Charlson } \\
\text { comorbidity score (\%) }\end{array}$ & $3(1-4)$ & $2(1-4)$ & $3(1-4)$ & $3(1-4)$ \\
\hline $\begin{array}{l}\text { Median (interquartile range) eGFR } \\
\left(\mathrm{mL} / \mathrm{min} / 1.73 \mathrm{~m}^{2}\right) \dagger\end{array}$ & $47.1(32.3-63.5)$ & $58.4(43.4-73.5)$ & $47.1(32.3-63.5)$ & $58.0(42.8-72.3)$ \\
\hline $\begin{array}{l}\text { Median (interquartile range) ejection } \\
\text { fraction (\%) }\end{array}$ & $29(20-33)$ & $25(20-30)$ & $29(20-33)$ & $25(20-30)$ \\
\hline $\begin{array}{l}\text { Median (interquartile range) serum BNP } \\
(\mathrm{pg} / \mathrm{mL})\end{array}$ & $1249(657-2258)$ & $681(304-1477)$ & $1249(657-2258)$ & $842(346-1560)$ \\
\hline Missing & $12,810(72)$ & $8989(78)$ & $12,810(72)$ & $786(72)$ \\
\hline $\begin{array}{l}\text { Median (interquartile range) serum } \\
\text { creatinine ( } \mathrm{mg} / \mathrm{dL} \text { ) }\end{array}$ & $1.3(1.0-1.8)$ & $1.2(1.0-1.5)$ & $1.3(1.0-1.8)$ & $1.2(1.0-1.5)$ \\
\hline Missing & $11488(64)$ & $114(1)$ & $11488(64)$ & $14(1)$ \\
\hline $\begin{array}{l}\text { Median (interquartile range) serum } \\
\text { sodium ( } \mathrm{mEq} / \mathrm{L})\end{array}$ & $138(136-141)$ & $139(137-141)$ & $138(136-141)$ & $139(137-141)$ \\
\hline Missing & $12032(67)$ & $157(1)$ & $12032(67)$ & $27(2)$ \\
\hline $\begin{array}{l}\text { Median (interquartile range) systolic } \\
\text { blood pressure ( } \mathrm{mm} \mathrm{Hg} \text { ) }\end{array}$ & $133(116-152)$ & $131(118-148)$ & $133(116-152)$ & $130(115-148)$ \\
\hline Missing & $9230(52)$ & $196(2)$ & $9230(52)$ & $19(2)$ \\
\hline \multicolumn{5}{|l|}{ Screening and prevention history: } \\
\hline Bone mineral density test $\ddagger$ & $677(8)$ & $373(12)$ & $677(8)$ & $34(11)$ \\
\hline Fecal occult blood test & $1111(6)$ & $876(8)$ & $1111(6)$ & $82(8)$ \\
\hline Influenza vaccine & $8423(47)$ & $6117(53)$ & $8423(47)$ & $547(50)$ \\
\hline
\end{tabular}


Table 1 (continued)

\begin{tabular}{|c|c|c|c|c|}
\hline \multirow[b]{2}{*}{ Characteristics } & \multicolumn{2}{|c|}{ Cohort 1 (ICD+heart failure registry population) } & \multicolumn{2}{|c|}{ Cohort 2 (heart failure registry population) } \\
\hline & No ICD ( $n=17853)$ & ICD ( $n=11573)$ & No ICD $(n=17853)$ & ICD (n=1089) \\
\hline Mammography† & $1472(17)$ & $909(30)$ & $1472(17)$ & $86(27)$ \\
\hline Pneumococcal vaccine & $1398(8)$ & $1076(9)$ & $1398(8)$ & $125(11)$ \\
\hline Prostate specific antigen test§ & $2622(28)$ & $2910(34)$ & $2622(28)$ & $262(34)$ \\
\hline
\end{tabular}

$\mathrm{COPD}=$ chronic obstructive pulmonary disease; eGRF=estimated glomerular filtration rate; $\mathrm{SNF}=$ skilled nursing facility; $\mathrm{BNP}=\mathrm{B}$ type natriuretic peptide. *Does not include non-melanoma skin cancer.

†eGFR was calculated according to the Chronic Kidney Disease Epidemiology Collaboration equation ${ }^{42}$ after discounting serum creatinine values by $5 \%$ to account for assay variability. ${ }^{43}$

łln women only.

§In men only. 
Table 2| Incidence of admission to a skilled nursing facility (SNF), hospital admission for hip fracture, and 30 day mortality in patients with or without implantable cardioverter-defibrillator (ICD) therapy

\begin{tabular}{|c|c|c|c|c|}
\hline & & No ICD & & ICD \\
\hline Event & Person years Events & $\begin{array}{c}\text { Incidence/1000 person years } \\
(95 \% \mathrm{Cl})\end{array}$ & Person years Events & $\begin{array}{l}\text { Incidence/1000 person years } \\
(95 \% \mathrm{Cl})\end{array}$ \\
\hline
\end{tabular}

Cohort 1 (ICD and heart failure registry

population):

\begin{tabular}{|c|c|c|c|c|c|c|}
\hline Hip fracture admission & 26819 & 467 & 17 (16 to 19$)$ & 24066 & 209 & $9(8$ to 10$)$ \\
\hline SNF admission & 19852 & 7037 & 354 (346 to 363$)$ & 21718 & 2438 & $112(108$ to 117$)$ \\
\hline Death in SNF or length of stay $>20$ days & 25662 & 1995 & $78(74$ to 81$)$ & 23814 & 556 & 23 (21 to 25$)$ \\
\hline 30 day mortality & 1337 & 2272 & $1699(1631$ to 1770$)$ & 945 & 156 & 165 (141 to 193$)$ \\
\hline \multicolumn{7}{|l|}{$\begin{array}{l}\text { Cohort } 2 \text { (heart failure registry } \\
\text { population): }\end{array}$} \\
\hline Hip fracture admission & 26819 & 467 & $17(16$ to 19$)$ & 2190 & 16 & 7 (4 to 12$)$ \\
\hline SNF admission & 19852 & 7037 & 354 (346 to 363 ) & 1934 & 258 & $133(118$ to 150$)$ \\
\hline Death in SNF or length of stay $>20$ days & 25662 & 1995 & 78 (74 to 81$)$ & 2154 & 63 & 29 (23 to 37 ) \\
\hline 30 day mortality & 1337 & 2272 & 1699 (1631 to 1770$)$ & 89 & 10 & 112 (58 to 199$)$ \\
\hline
\end{tabular}


Table 3| Hazards of admission to a skilled nursing facility (SNF), hospital admission for hip fracture, and 30 day mortality in patients with or without an implantable cardioverter-defibrillator (ICD)

\begin{tabular}{|c|c|c|c|}
\hline \multirow[b]{2}{*}{ Event } & \multicolumn{3}{|c|}{ Hazard ratio $(95 \% \mathrm{Cl})$} \\
\hline & Unadjusted & Age and sex adjusted & Multivariable adjusted $^{*}$ \\
\hline \multicolumn{4}{|l|}{$\begin{array}{l}\text { Cohort } 1 \text { (ICD and heart failure registry } \\
\text { population): }\end{array}$} \\
\hline Non-traumatic hip fracture & 0.51 (0.43 to 0.59$)$ & 0.77 (0.64 to 0.92$)$ & $0.84(0.66$ to 1.05$)$ \\
\hline SNF admission & $0.39(0.37$ to 0.41$)$ & $0.53(0.50$ to 0.55$)$ & $0.71(0.67$ to 0.76$)$ \\
\hline Death in SNF or length of stay $>20$ days & $0.36(0.33$ to 0.39$)$ & 0.50 (0.45 to 0.56$)$ & $0.69(0.60$ to 0.80$)$ \\
\hline 30 day mortality & $0.10(0.09$ to 0.12$)$ & $0.12(0.10$ to 0.15$)$ & $0.20(0.17$ to 0.24$)$ \\
\hline \multicolumn{4}{|l|}{ Cohort 2 (heart failure registry population): } \\
\hline Non-traumatic hip fracture & $0.42(0.23$ to 0.67$)$ & $0.63(0.36$ to 1.01$)$ & $0.53(0.31$ to 0.92$)$ \\
\hline SNF admission & $0.47(0.41$ to 0.53$)$ & 0.60 (0.53 to 0.68$)$ & $0.68(0.60$ to 0.78$)$ \\
\hline Death in SNF or length of stay $>20$ days & 0.45 (0.34 to 0.57$)$ & 0.61 (0.47 to 0.78$)$ & $0.78(0.53$ to 1.15$)$ \\
\hline 30 day mortality & 0.07 (0.03 to 0.12$)$ & 0.09 (0.04 to 0.15$)$ & 0.12 (0.06 to 0.22$)$ \\
\hline
\end{tabular}

${ }^{*}$ Adjusted for all covariates listed in web extra appendix III, including clinical variables such as ejection fraction, systolic blood pressure, sodium level, serum B type natriuretic peptide, and estimated glomerular filtration rate. 


\section{Figures}

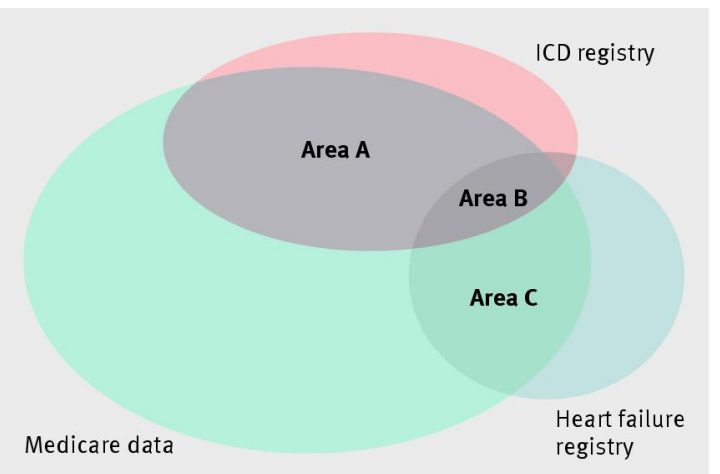

Fig 1 Conceptual relation of data sources and schematic presentation of cohort 1 (implantable converter-defibrillator (ICD)+heart failure registry population) and cohort 2 (heart failure registry population). Cohort 1 consists of patients receiving ICD therapy from overlapping area between ICD registry and Medicare data (area $A+B$ ) and patients not receiving ICD therapy from overlapping area between heart failure registry and Medicare data but no overlap with ICD registry (area C). Cohort 2 consists of patients receiving ICD therapy from overlapping area between the heart failure registry, ICD registry, and Medicare data (area B) and patients not receiving ICD therapy from area C
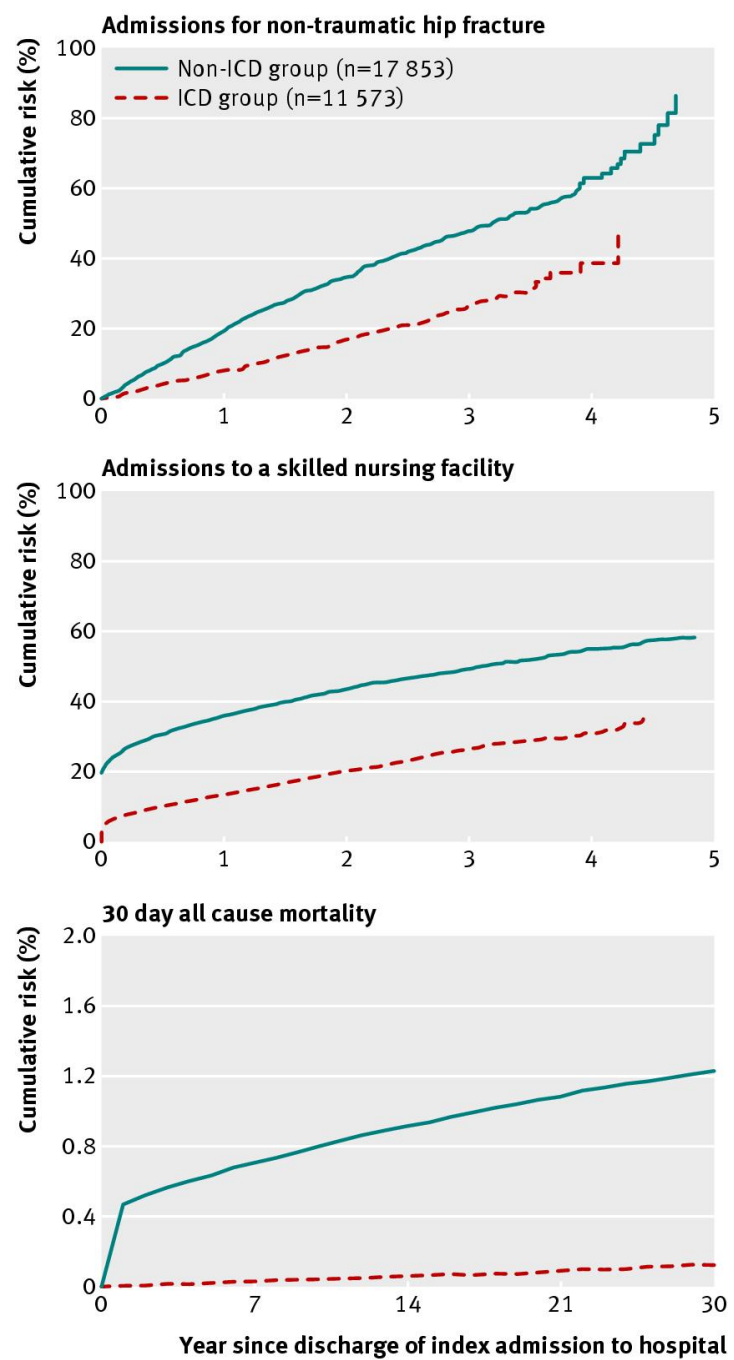

Fig 2 Crude survival curves of patients receiving and not receiving implantable converter-defibrillator (ICD) therapy in cohort 1 for admissions for non-traumatic hip fracture, admissions to a skilled nursing home, and 30 day all cause mortality 